\title{
Calculation of transfer functions for volume rendering of breast tomosynthesis imaging
}

Mota, Ana M., Clarkson, Matthew, Orvalho, Lurdes, Almeida, Pedro, Matela, Nuno

Ana M. Mota, Matthew J. Clarkson, Lurdes Orvalho, Pedro Almeida, Nuno Matela, "Calculation of transfer functions for volume rendering of breast tomosynthesis imaging," Proc. SPIE 11513, 15th International Workshop on Breast Imaging (IWBI2020), 1151327 (22 May 2020); doi: $10.1117 / 12.2559932$

Event: Fifteenth International Workshop on Breast Imaging, 2020, Leuven, Belgium 


\title{
Calculation of transfer functions for Volume Rendering of Breast Tomosynthesis imaging
}

\author{
Ana M. Mota*a, Matthew J. Clarkson ${ }^{\mathrm{b}}$, Lurdes Orvalho ${ }^{\mathrm{c}}$, Pedro Almeida ${ }^{\mathrm{a}}$, Nuno Matela ${ }^{\mathrm{a}}$ \\ anstituto de Biofísica e Engenharia Biomédica, Faculdade de Ciências, Universidade de Lisboa, \\ Portugal; ${ }^{\mathrm{b}}$ Department of Medical Physics and Biomedical Engineering and the Centre for Medical \\ Image Computing, University College London, London, UK; ${ }^{\mathrm{C} H o s p i t a l}$ da Luz, Lisboa, Portugal
}

\begin{abstract}
Slice by slice visualization of Digital Breast Tomosynthesis (DBT) data is time consuming and can hamper the interpretation of lesions such as clusters of microcalcifications. With a visualization of the object through multiple angles, 3D volume rendering (VR) provides an intuitive understanding of the underlying data at once. 3D VR may play an important complementary role in breast cancer diagnosis. Transfer functions (TFs) are a critical parameter in VR and finding good TFs is a major challenge. The purpose of this work is to study a methodology to automatically generate TFs that result in appropriate and useful VR visualizations of DBT data.

For intensity-based TFs, intensity histograms were used to study possible relationships between statistics and critical intensity values in DBT data. The mean of each histogram has proved to be a valid option to automatically calculate those critical values that define these functions. At this stage, eight visualizations were obtained by combining several opacity/color intensity-based functions. Considering the gradient, ten visualizations were obtained. Nine of the ten TFs were constructed considering the peaks of gradient magnitude histograms. The tenth function was a simple linear ramp. Finally, three intensity-based and three gradient-based functions were selected and simultaneously used. This resulted in nine final VR visualizations taking both information into account.

The studied approach allowed an automatic generation of opacity/color TFs based on scalar intensity and gradient magnitude histograms. In this way, the preliminary results obtained with this methodology are very encouraging about creating an adequate visualization of DBT data by VR.
\end{abstract}

Keywords: Digital breast tomosynthesis, volume rendering, 3D visualization, transfer function

\section{INTRODUCTION}

Digital Breast Tomosynthesis (DBT) has been consolidating its position as a technique to replace 2D Digital Mammography (DM) in both screening and clinical environment ${ }^{1-4}$. So far, DBT data is displayed one slice at a time or sequentially as a continuous cine loop. This procedure can lead to difficult interpretation of microcalcification clusters, which can be spread across several slices ${ }^{5}$. In addition, the mean reading time doubles for DBT compared with DM examination $^{6-8}$. The time required for each DBT data set evaluation is crucial both in clinical and screening environments, directly influencing the number of examinations interpreted ${ }^{8}$.

A different type of visualization, such as 3D volume rendering (VR), may play an important complementary role in breast cancer diagnosis 9 . 3D VR is the process of creating realistic computer-generated images of a 3D scene, yielding a true depth perception ${ }^{10}$. With a visualization of the object through multiple angles, one of the advantages of 3D VR is to provide an intuitive understanding of the underlying data at once. Transfer functions (TFs) are a critical parameter in VR. They define how much and which data are visible by assigning opacity and color to the intensity and/or gradient magnitude values. To provide useful information about the volume data, TFs must ensure a balance between what is made transparent and what is considered to be of interest and must be opaque. Finding good TFs is one of the major challenges in volume visualization. It is time consuming and accomplished by a frustrating trial and error process ${ }^{11-13}$. Automatic and semi-automatic generation of TFs is the ultimate goal in many applications since it enables a more widespread use of $\mathrm{VR}^{11,14,15}$.

There are some works that mention a few aspects of 3D VR for $\mathrm{DBT}^{16-18}$ and its importance to detect clusters of microcalcifications ${ }^{19}$. However, there are currently no established methods or conclusions on acceptable TFs to provide 
adequate 3D VR visualization of DBT data. For this reason, opacity and color values were assigned to the intensity and gradient magnitude values of the data ${ }^{20}$ and an innovative methodology to automatically generate TFs which allow an adequate 3D visualization by VR of DBT data is presented.

\section{METHODS}

\subsection{Data visualization}

Twenty DBT data sets of anonymous patients from a clinical facility database were selected (Hospital da Luz S.A., Lisbon, Portugal). The heterogeneity of the sample was taken into account: there are ten cases acquired with mediolateral oblique view and ten with craniocaudal view, 50\% are from right breasts and 50\% from left breasts. All breast density levels, as well as all diagnostic classifications (based on the criteria of the American College of Radiology's Breast Imaging Reporting and Data System - BI-RADS) are included in this study.

The visualization software was developed in $\mathrm{C}++$ using the Visualization Toolkit library (VTK) version 7.1.0. ${ }^{21}$. For a better visualization quality, voxels were made isotropic (with dimensions $0.085 \times 0.085 \times 0.085 \mathrm{~mm}^{3}$ ) using the Lanczos function available in VTK (used by default) ${ }^{22}$. 3D VR was obtained with composite technique.

\subsection{Generation of scalar intensity-based TFs}

For each of the twenty cases, two regions were selected and the respective intensity values recorded. Region 1 is related to adipose tissue (similar to background) and region 2 refers to high intensity materials, such as microcalcifications. As the twenty cases comprehend all different levels of BI-RADS diagnosis, in cases with calcifications, region 2 includes data both on benign and malignant calcifications.

$a 1$ and $b 1$ stand for the intensity value of region 1 and 2, respectively. Voxels with scalar intensity values below $a 1$ are considered totally transparent, above $b 1$ are totally opaque and with intermediate values correspond to a linear relationship between intensity and opacity/color (Figure 1).

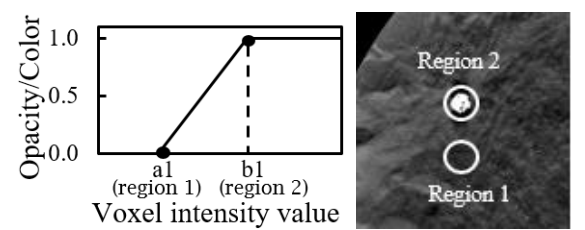

Figure 1 (a) - 1D linear ramp transfer function used to map voxel intensity values to opacity and colour. (b) Zoom in on the region 1 and region 2 whose intensities correspond to a1 and b1, respectively.

Three intensity histogram statistics - mean, maximum and skewness - were calculated for each case. The relationships between $a l$ and $b l$ and the intensity histogram statistics were studied to obtain possible expressions to estimate $a l$ and $b 1$, having knowledge of the statistics. Additionally, two other intensity values $-a 2$ and $b 2$ - were calculated in order to achieve more distinct visualizations (Eq. (1) and Eq. (2)). Taking into account different combinations of this critical values, eight visualizations were obtained.

$$
\begin{aligned}
& a 2=a 1+\frac{b 1-a 1}{2} \\
& b 2=b 1+\frac{b 1-a 1}{2}
\end{aligned}
$$

\subsection{Generation of gradient magnitude-based TFs}

Gradient magnitude is a useful second dimension because it measures how quickly values are changing in the image space. Breast tissues are relatively homogeneous, so their gradient magnitudes are low. The peaks in the gradient histograms correspond to sudden intensity changes, which means, boundaries between two materials ${ }^{13}$.

Here, to only have one degree of freedom (gradient), the influence of intensity-based TFs was kept neutral (opacity and color values were kept at 1 for all scalar intensity values). For each DBT case, three peaks in the gradient magnitude histogram were found. Based on these peaks, nine opacity TFs were analyzed. In addition, one function independent from the peaks, with a shape of a linear ramp crossing all magnitude gradient values, was also considered. 


\subsection{TFs based on scalar intensity and gradient magnitude}

By combining some results from section 2.2 and section 2.3, nine different final visualizations by VR were obtained taking into account both information (scalar intensity and gradient magnitude).

\section{RESULTS}

For all the twenty cases, it was observed that $a 1$ and $b 1$ measured values were at the same position in relation to the respective histogram. Based on this sample, linear regressions were modelled to study the relationship between $a l$ and $b l$ and some statistics (mean, maximum and skewness) of each intensity histogram. Results are presented in Figure 2.
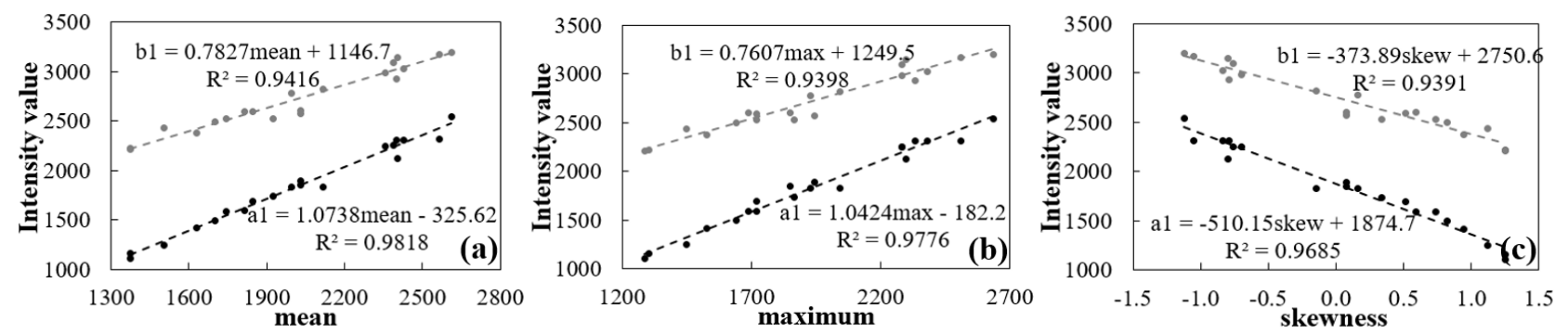

Figure 2. Linear regressions obtained between $a l$ and $b 1$ and (a) mean, (b) maximum and (c) skewness of histogram intensity values, calculated for each clinical case. Coefficients of determination $\left(\mathrm{R}^{2}\right)$ and equations for the estimation of $a 1$ and $b l$ based on each statistic are also shown.

The eight combinations of opacity and color TFs based on scalar intensity values $(a 1, a 2, b 1$ and $b 2)$ and respective 2D displays of VR results at $0^{\circ}$ are shown in Figure 3. On the other hand, the VR results obtained with the ten gradient-based TFs, keeping the intensity-based TFs as neutral, are presented in Figure 4.

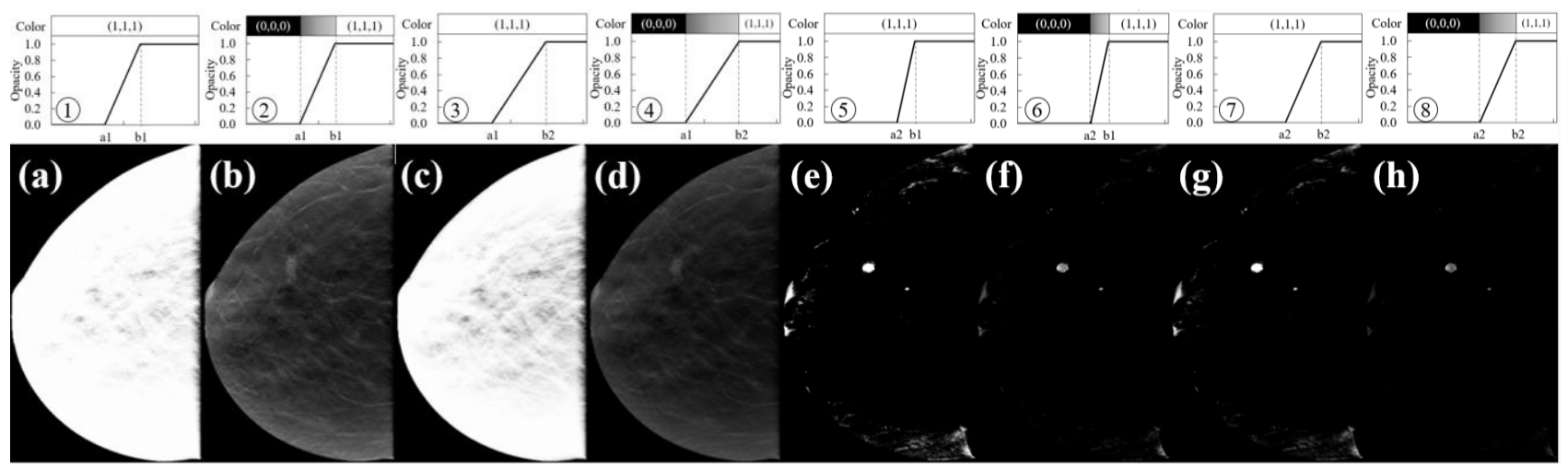

Figure 3. 2D displays of VR visualizations at $0^{\circ}$ obtained with each combination of intensity-based TFs by varying the color and opacity within the values of $a 1, a 2, b 1$ and $b 2$. 

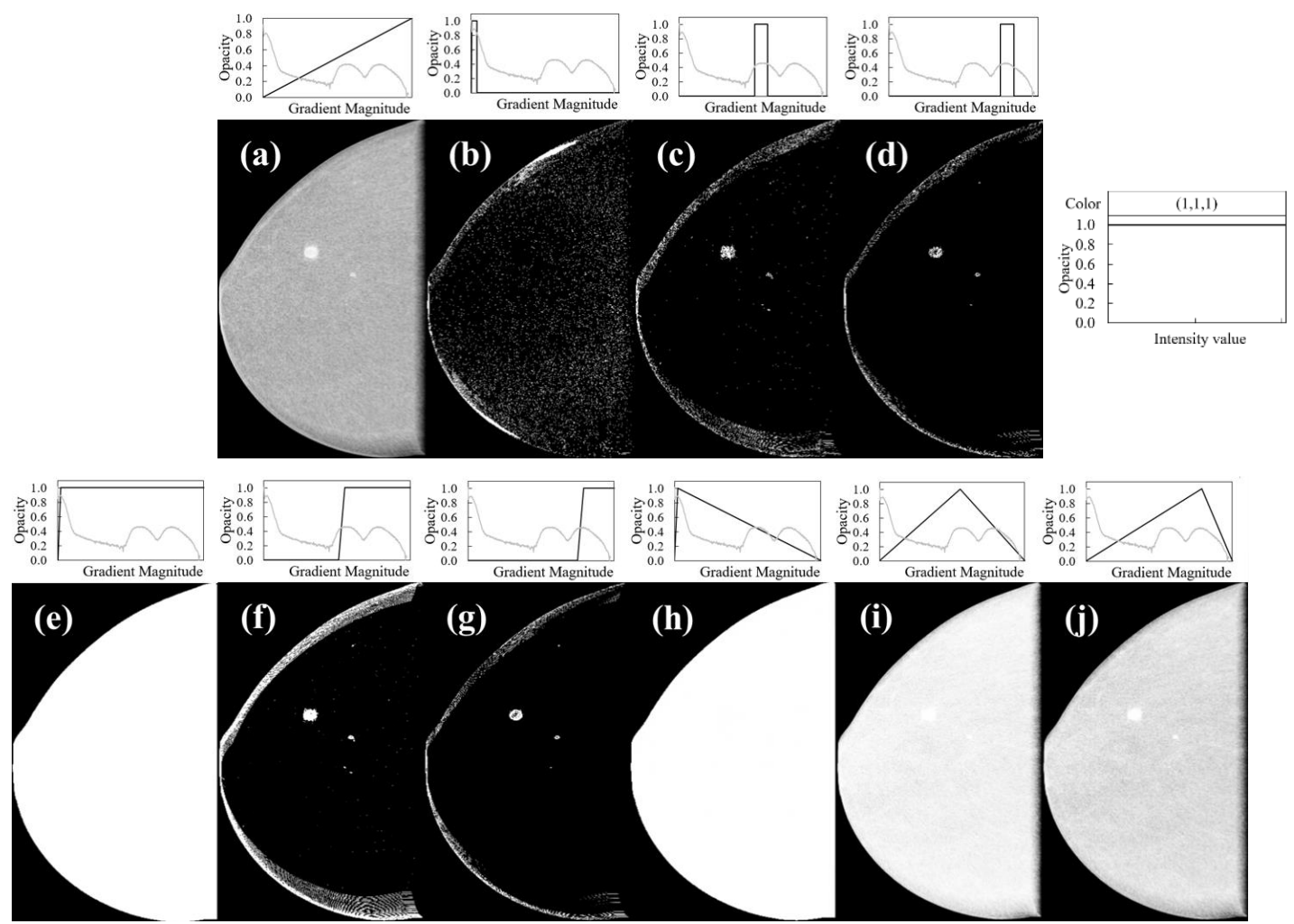

Figure 4. 2D displays of VR visualizations at $0^{\circ}$ obtained with the ten gradient-based TFs: linear ramp (a), square shaped centered on each peak (b,c,d), ramp-shaped around each peak (e,f,g) and triangular shaped centered on each peak (h,i,j). Top right corner: Opacity and color values were kept at 1 for all scalar intensities.

In order to merge the information provided by the intensity-based functions (Figure 3) with the gradient-functions (Figure 4), some visualizations of both groups have been selected and combined. From Figure 3, it is possible to group three similar visualizations: (1) Figure 3(a) and (c); (2) Figure 3(b) and (d); (3) Figure 3(e-h). From the first group, the combination of functions in Figure 3(a) was selected because it allowed to keep more information about all tissues. From the second one, to have an intermediate visualization, functions of Figure 3(b) were chosen. Of the third group, functions presented in Figure 3(f) were elected because they emphasize important structures of interest for breast cancer detection, such as calcifications, in a balanced way. Regarding VR resulting from the assignment of opacity to gradient magnitude (Figure 4), there are 7 out of 10 that can be immediately excluded: Figure 4(b-h). TFs corresponding to visualizations of Figure 4(b-d) and Figure 4(f-g) remove a significant amount of information. On the other hand, functions from Figure 4(e) and (h) make too much information opaque (which means that conjugated with intensity-based functions, these gradient information have a minor impact on the final VR). In this way, by combining TFs from Figure 3(a), (b) and (f) with those of Figure 4(a), (i) and (j), nine visualizations are obtained as in Figure 5. 


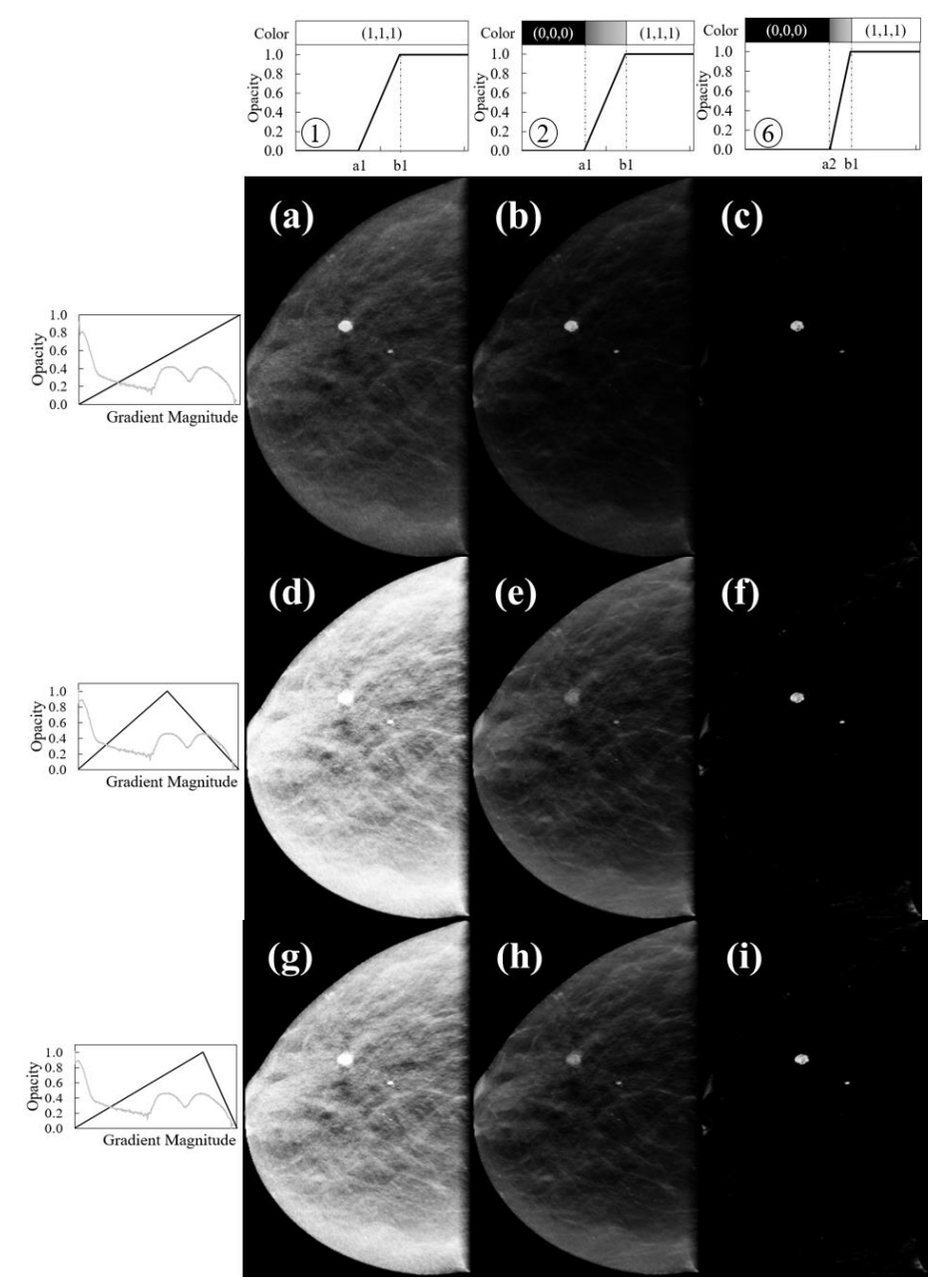

Figure 5. 2D displays of VR visualizations at $0^{\circ}$ considering intensity and gradient magnitude functions selected. 1 st, $2 \mathrm{nd}$ and 3rd rows were obtained using the gradient-based functions: linear ramp and triangular-shaped centered on peak 2 and 3 , respectively. 1st, 2nd and 3rd columns were obtained using the scalar intensity-based functions: 1, 2 and 6, respectively.

\section{DISCUSSION AND CONCLUSION}

A consistent and reproducible methodology for automatic determination of TFs allowing an adequate 3D visualization by VR of DBT data was presented. Opacity/color TFs based on scalar intensity and gradient magnitude were tested separately and together and the results were visualized through composite technique.

Regardless of breast density, all intensity histograms presented the same shape, i.e., neglecting the zero intensity peak corresponding to the black background, a single peak was observed. The measured values for $a l$ and $b 1$ were at the same position in relation to the respective histogram. From Figure 2, the statistic which presented the best option for automatically estimate $a l$ and $b 1$ from the data was the mean, with the highest $\mathrm{R}^{2}$ simultaneously for the regression with $a 1$ and $b 1$ (0.9818 and 0.9416, respectively). With the eight VR images observed based on different values of $a l, a 2, b 1$ and $b 2$ (Figure 3), we can see that as the TFs move to the right, the tissues with lower values of intensity become transparent, emphasizing only those of higher intensities, as expected.

As for the intensity, all gradient magnitude histograms presented the same shape for all the twenty cases, independent of breast density. Three main peaks were observed. In a general way, TFs based on gradient magnitude are closely related to the gradient histogram peaks (Figure 4).The obtained VR images using only scalar intensity TFs (Figure 3 (a), (b) and (f)) can be compared with those using scalar intensity plus gradient magnitude TFs (1st, 2nd and 3rd column of Figure 5). The general appearance of the latter was determined essentially by TFs based on scalar intensity while the gradientbased TFs improved the definition between regions with different intensities. 
Taking into account the preliminary results presented, we conclude that adequate visualization of DBT data by VR can be automatically achieve.

In the future, the impact of VR on detection performance of radiologists and visualization of masses and architectural distortions should be considered through a large and controlled clinical study with 3D VR using DBT data.

Acknowledgments. This work was supported by Universidade de Lisboa (PhD grant) and Fundação para a Ciência e Tecnologia - Portugal (Grant No. SFRH/BD/135733/2018 and FCT-IBEB Strategic Project UID/BIO/00645/2013).

\section{REFERENCES}

[1] Gennaro, G., Toledano, A., di Maggio, C. et al., "Digital breast tomosynthesis versus digital mammography: a clinical performance study" Eur Radiol, 20(7), 1545-1553 (2010).

[2] Bonafede, M. M., Kalra, V. B., Miller, J. D. et al., "Value analysis of digital breast tomosynthesis for breast cancer screening in a commercially-insured US population" ClinicoEconomics and Outcomes Research: CEOR, 7, 53-63 (2015).

[3] Gao, Y., Babb, J. S., Toth, H. K. et al., "Digital Breast Tomosynthesis Practice Patterns Following 2011 FDA Approval: A Survey of Breast Imaging Radiologists" Acad Radiol, 24(8), 947-953 (2017).

[4] Destounis, S., Santacroce, A., and Arieno, A., "DBT as a Screening Tool and a Diagnostic Tool" Current Breast Cancer Reports, 9(4), 264-271 (2017).

[5] Samala, R. K., Chan, H.-P., Lu, Y. et al., "Digital breast tomosynthesis: Computer-aided detection of clustered microcalcifications on planar projection images" Phys Med Biol, 59(23), 7457-7477 (2014).

[6] Good, W. F., Abrams, G. S., Catullo, V. J. et al., "Digital breast tomosynthesis: a pilot observer study" AJR Am J Roentgenol, 190(4), 865-9 (2008).

[7] Gur, D., Abrams, G. S., Chough, D. M. et al., "Digital breast tomosynthesis: observer performance study" AJR Am J Roentgenol, 193(2), 586-91 (2009).

[8] Caumo, F., Zorzi, M., Brunelli, S. et al., "Digital Breast Tomosynthesis with Synthesized Two-Dimensional Images versus Full-Field Digital Mammography for Population Screening: Outcomes from the Verona Screening Program" Radiology, 287(1), 37-46 (2018).

[9] Venson, J. E., Albiero Berni, J. C., Edmilson da Silva Maia, C. et al., "A Case-Based Study with Radiologists Performing Diagnosis Tasks in Virtual Reality" Stud Health Technol Inform., 245, 244-248. (2017).

[10] Suetens, P., [Medical image analysis] Cambridge University Press, New York(2009).

[11] Kindlmann, G., and Durkin, J. W., "Semi-automatic generation of transfer functions for direct volume rendering". 79-86.

[12] Kindlmann, G., "Transfer functions in direct volume rendering: Design, Interface, Interaction”, (September 2017), http://www.cs.utah.edu/ gk/papers/sig02-TF-notes.pdf (November 2019).

[13] Kniss, J. K., G.; Hansen, C; [Visualization Handbook] Academic Press, Multi-Dimensional Transfer Functions for Volume Rendering (2004).

[14] Correa, C. D., and Ma, K. L., "Visibility Histograms and Visibility-Driven Transfer Functions" IEEE Transactions on Visualization and Computer Graphics, 17(2), 192-204 (2011).

[15] Ma, B., and Entezari, A., "Volumetric Feature-Based Classification and Visibility Analysis for Transfer Function Design" IEEE Transactions on Visualization and Computer Graphics, PP(99), 1-1 (2017).

[16] Alyassin, A. M., "Automatic transfer function generation for volume rendering of high-resolution x-ray 3D digital mammography images". 4681, 11.

[17] Alyassin, A. M., Eberhard, J. W., Claus, B. E. H. et al., [3D Visualization of X-ray Tomosynthesis Digital Mammography Data: Preference Study] Springer Berlin Heidelberg, Berlin, Heidelberg(2003).

[18] Dharanija, R., and Rajalakshmi, T., "A Conjunct Analysis for Breast Cancer Detection by Volume Rendering of Low Dosage Three Dimensional Mammogram". 1361-1365.

[19] Jerebko, A., Engel, K., Hofmann, C. et al., "3D rendering methods for visualization of clusters of calcifications in digital breast tomosynthesis: a feasibility study".

[20] Ljung, P., Krüger, J., Groller, E. et al., "State of the Art in Transfer Functions for Direct Volume Rendering" Computer Graphics Forum, 35(3), 669-691 (2016).

[21] Schroeder, W., Martin, K., and Lorensen, B., [The Visualization Toolkit: An Object-oriented Approach to 3D Graphics] Kitware, USA(2006). 
[22] VTK - Interpolators, "Visualization Toolkit - VTK - Interpolators", https://vtk.org/Wiki/VTK/Image_Interpolators (November 2019).

Proc. of SPIE Vol. $115131151327-7$

Downloaded From: https://www.spiedigitallibrary.org/conference-proceedings-of-spie on 26 Jul 2020 Terms of Use: https://www.spiedigitallibrary.org/terms-of-use 